\title{
Supplementary file for - A Distributed Epigenetic Shape Formation and Regeneration Algorithm for a Swarm of Robots
}

\author{
Rahul Shivnarayan Mishra \\ Indian Institute of Technology \\ Guwahati \\ rahulm@nvidia.com
}

\author{
Tushar Semwal \\ Indian Institute of Technology \\ Guwahati \\ t.semwal@iitg.ac.in
}

\author{
Shivashankar B. Nair \\ Indian Institute of Technology \\ Guwahati \\ sbnair@iitg.ac.in
}

ACM Reference Format:

Rahul Shivnarayan Mishra, Tushar Semwal, and Shivashankar B. Nair. 2018. Supplementary file for - A Distributed Epigenetic Shape Formation and Regeneration Algorithm for a Swarm of Robots. In Proceedings of ACM Conference (Conference'17). ACM, New York, NY, USA, 3 pages. https://doi. org/10.1145/nnnnnnn.nnnnnnn

This file contains information on the various techniques which have been proposed and implemented as a part of this work.

\section{SKEW COORDINATE SYSTEM}

The step by step graphical visualization to understand the generation of skewed bitmap is shown in Fig. 1. The associated pseudocode for the same is illustrated in Algorithm 1.

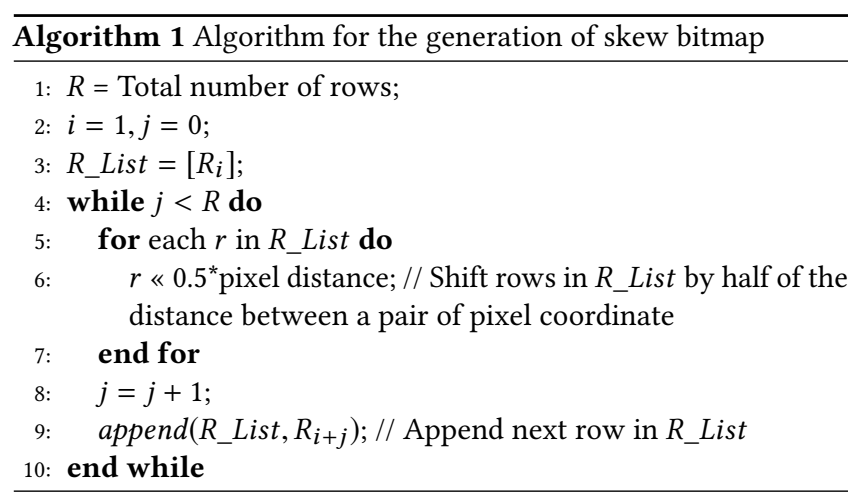

\section{GENERATION OF SCALED BITMAP}

The steps to generate a scaled bitmap are shown in Fig. 2.

\footnotetext{
Permission to make digital or hard copies of all or part of this work for personal or classroom use is granted without fee provided that copies are not made or distributed for profit or commercial advantage and that copies bear this notice and the full citation on the first page. Copyrights for components of this work owned by others than ACM must be honored. Abstracting with credit is permitted. To copy otherwise, or republish, to post on servers or to redistribute to lists, requires prior specific permission and/or a fee. Request permissions from permissions@acm.org.

Conference'17, fuly 2017, Washington, DC, USA

(c) 2018 Association for Computing Machinery. ACM ISBN 978-x-xxxx-xxxx-x/YY/MM...\$15.00

https://doi.org/10.1145/nnnnnnn.nnnnnnn
} 


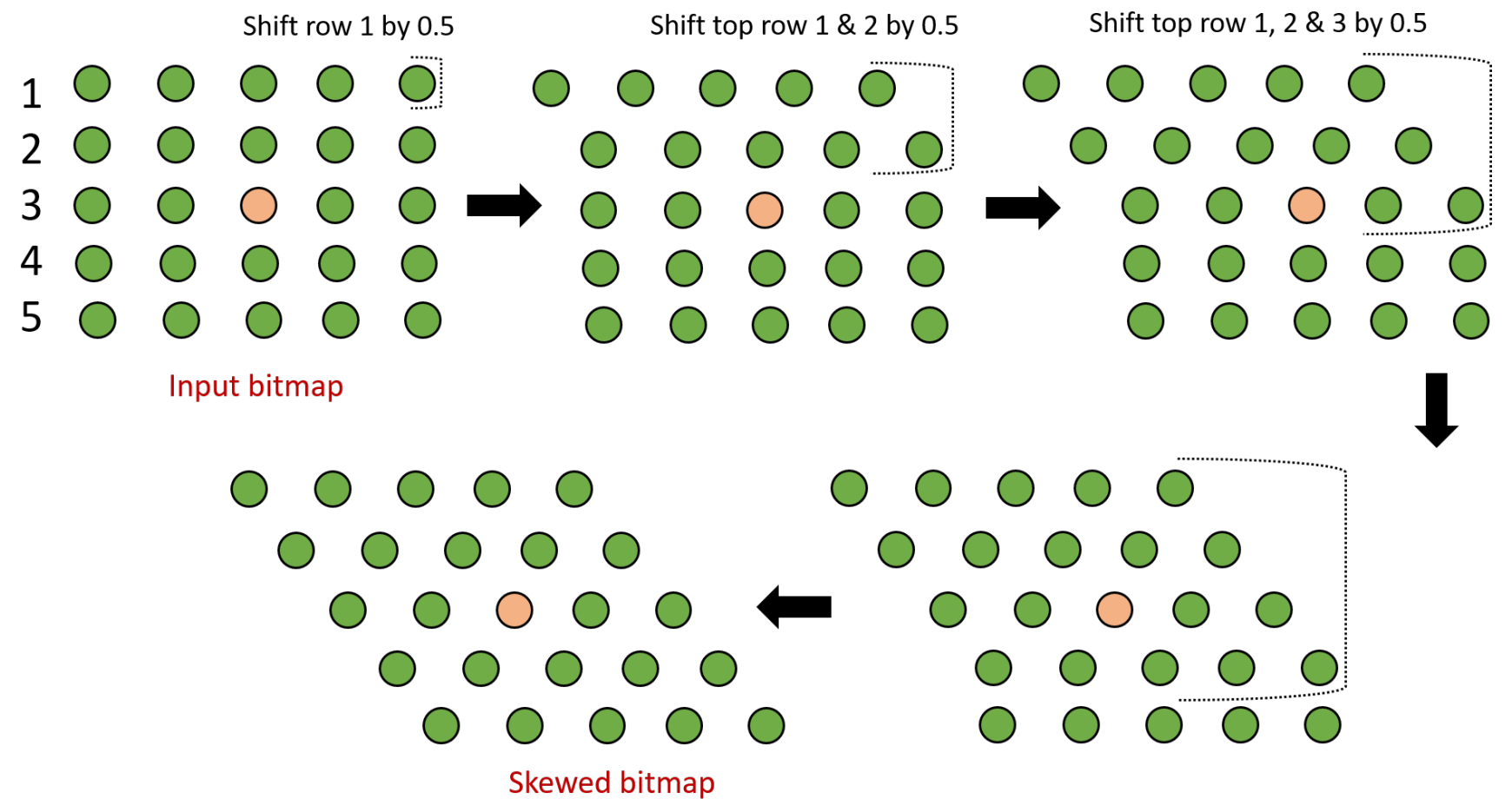

Figure 1: Steps to generate a skewed bitmap from an input bitmap in square Cartesian system 


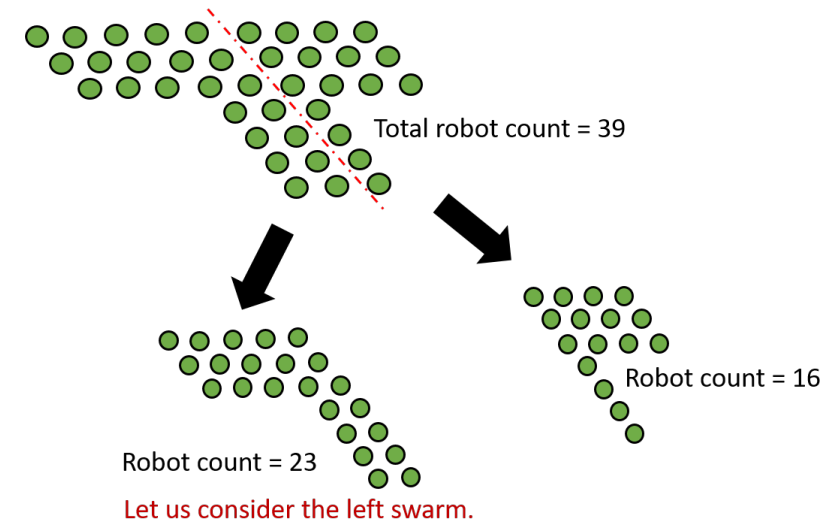

(a)

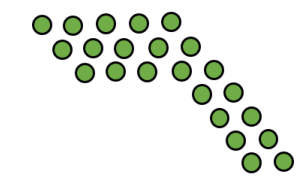

Now the leader needs to generate a scaled ' $\mathrm{T}$ ' shape with the remaining 23 robots.

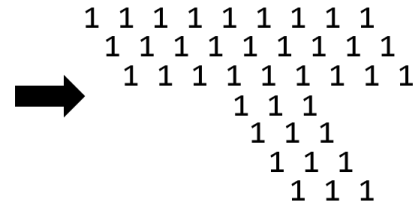

This bit cannot be removed as it will cause this bit to have less than two neighbours.

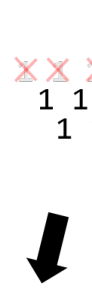

The obvious and easiest way to generate
a scaled image is to remove from the original
bitmap, as many bits as the number of robots
which are defunct.

The leader begins from the top left bit and starts removing $1 \mathrm{~s}$ from the periphery of the bitmap.

We have chosen to remove $1 \mathrm{~s}$ in a

Clockwise direction from the periphery of the bitmap, provided that the removal of a 1

does not cause any other 1 to have less than

two neighbouring $1 \mathrm{~s}$.

Counter-clockwise direction can also be chosen.

(b)

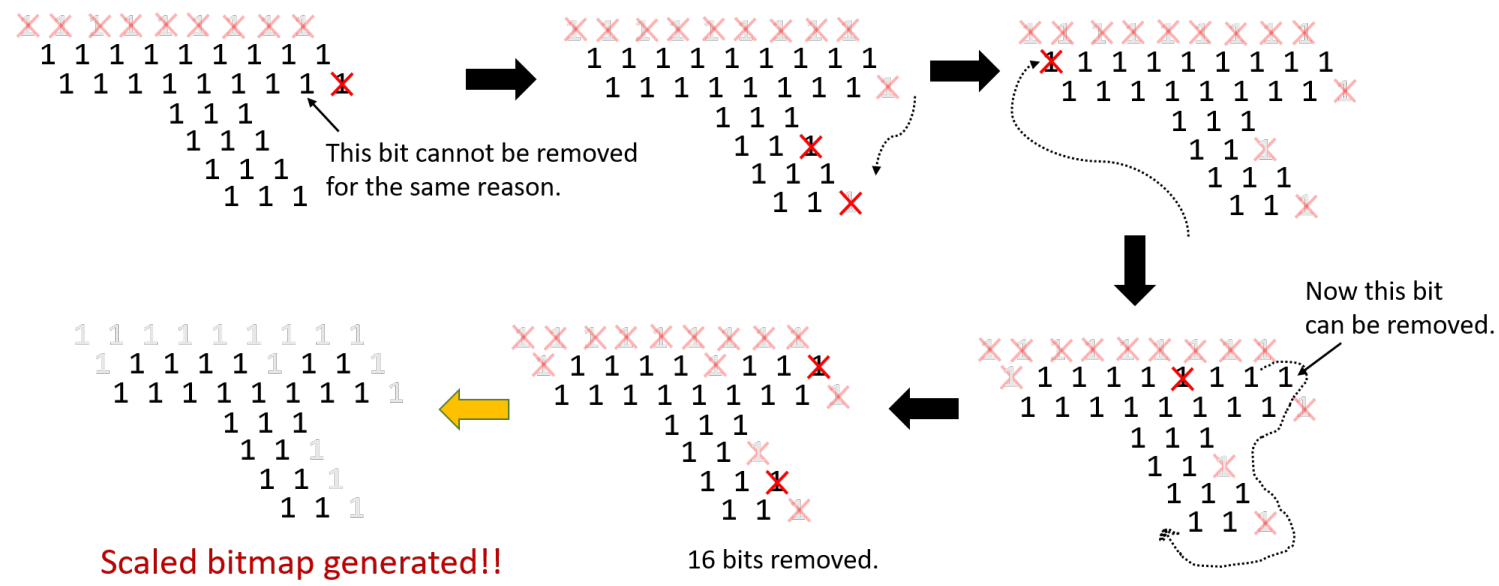

(c)

Figure 2: Generation of a scaled bitmap 